\title{
Chirurgische Behandlung schwer Brandverletzter
}

OP-ABLAUF Die Behandlung von schwer brandverletzten Patienten ist komplex und verlangt nach einer hohen Versorgungsqualität. Dr. A. Rahmanian-Schwarz und Operationsschwester Irene Jantzen aus dem Brandverletztenzentrum der Berufsgenossenschaftlichen Unfallklinik Tübingen geben einen Überblick über die wichtigsten Methoden der chirurgischen Versorgung.

\section{Planung und Vorbereitung}

Die Vorbereitung der Operation eines Verbrannten richtet sich nach der Verbrennungstiefe, die in 3 Grade eingeteilt wird:

Grad 1 - 2a: Rötung und Schwellung der Haut (Grad 1) mit Blasenbildung (Grad 2a). Hier ist keine OP notwendig, da aus der noch unverletzten tiefen Schicht des Corium Zellen einsprießen und eine Spontanheilung ohne Narbenbildung erfolgt. Der Verbrannte wird im Verbrennungsbad mit Bürsten ( $\rightarrow$ Abb. 1 ) oder Versajet tangential nekrektomiert und erhält einen temporären Hautersatz, bis sich die Haut regeneriert und der Verband sich ablöst.
Grad 2b - 3: Ab einer tiefen Schädigung des Corium (Grad 2b) oder Schädigung des subcutanen Fettgewebes (Grad 3) ist keine Spontanheilung mehr möglich, es muss operiert werden. Nach einem BürstenDebridement im Verbrennungsbad werden die Wunden beurteilt und das weitere operative Vorgehen geplant. Hierbei gibt es zwei Vorgehensweisen: Die komplette Exzision, bei der in einem Eingriff die gesamten tiefgradig verbrannten Areale entfernt und temporär gedeckt werden, oder die Frühexzision, bei der alle zwei Tage maximal 20\% der verbrannten Fläche abgetragen und definitiv gedeckt werden.
Wir wenden hauptsächlich drei operative Techniken an:

- Escheratomie: Bei zirkulären Verbrennungen Grad 2b und 3 werden mit der elektrischen Nadel Entlastungsschnitte gesetzt, um ein Kompartmentsyndrom zu verhindern ( $\rightarrow$ Abb. 2). Der Schnitt wird temporär mit Schweinehaut gedeckt.

- Tangentiale Nekrektomie: Bei Verbrennungen Grad 2b werden mit Versajet, Dermatom, Weck- oder Humby-Messer Epidermis und Corium entfernt. Ist eine definitive Deckung mit Eigenhaut nicht möglich, wird ein temporärer
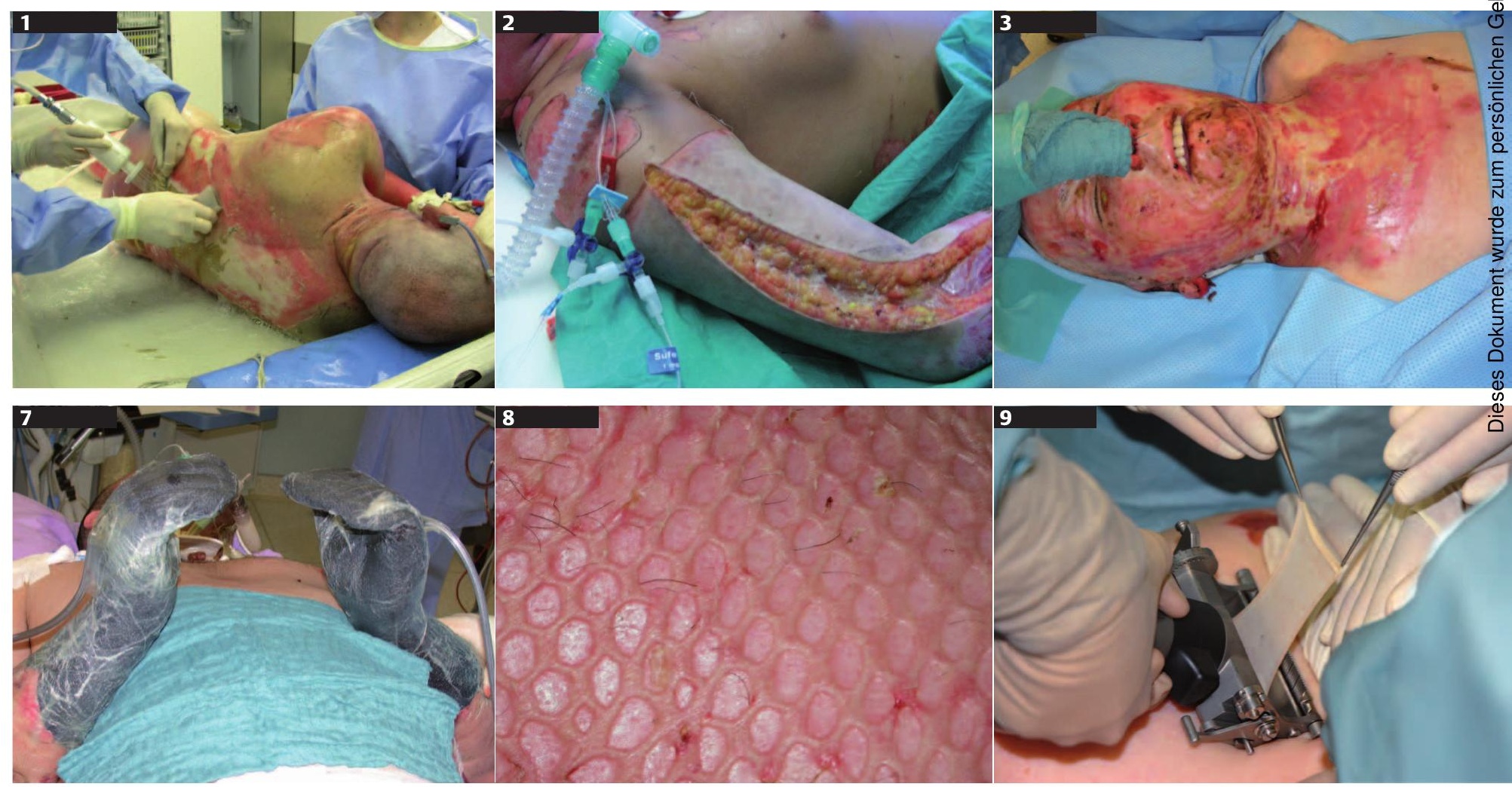


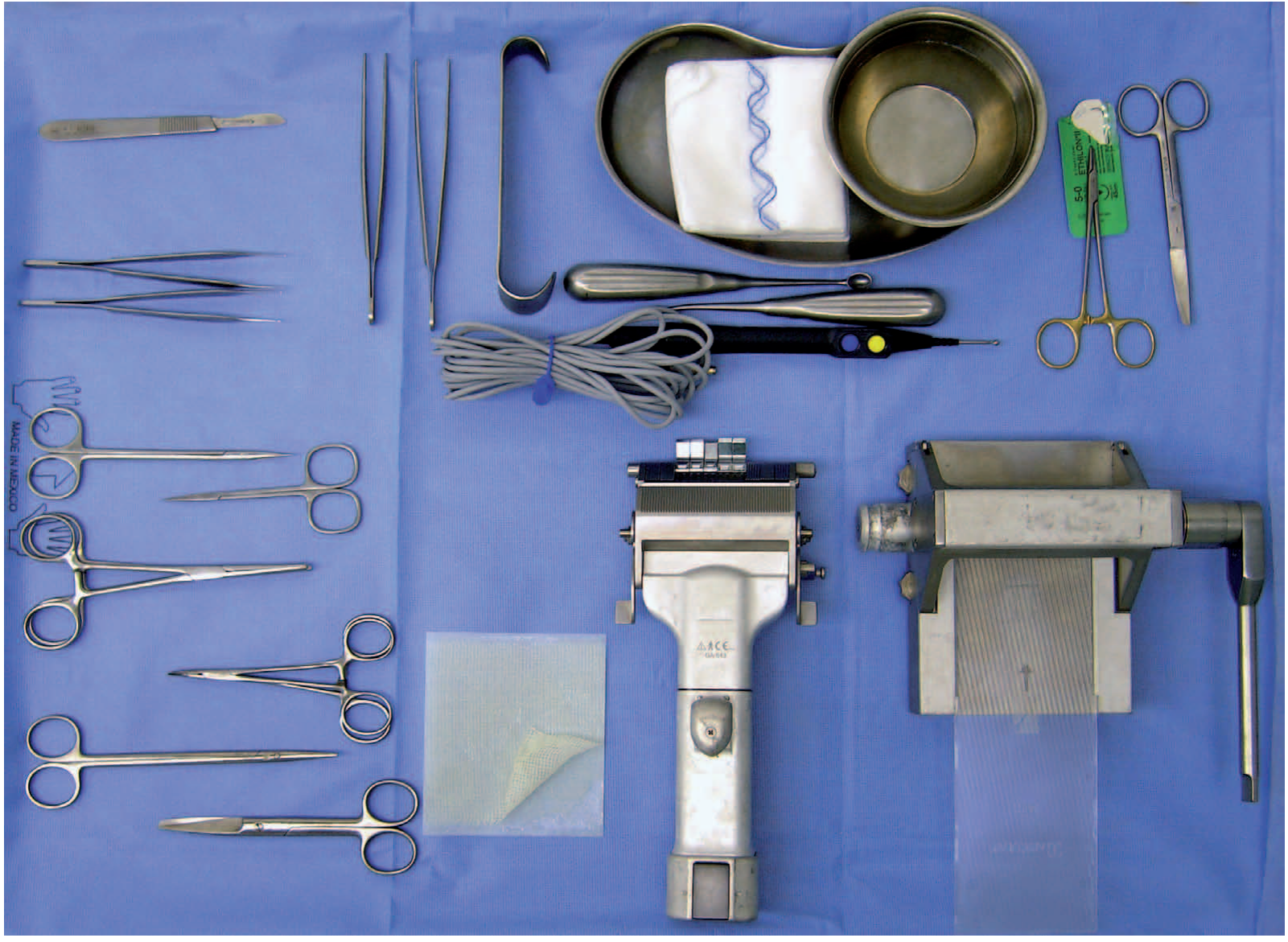

\section{INSTRUMENTE}

Links von oben nach unten:

- 1 10-er Skalpell

- 2 Adsonpinzetten chirurgisch

- 1 feine Präparierschere

- 1 feine Schere spitz-gerade

- 2 Kocher

- 2 Klemmchen scharf

- 1 Fadenschere

- 1 Materialschere

Oben von links nach rechts:

- 2 Pinzetten atraumatisch

- 1 Roux-Haken

- 1 Nierenschale mit Kompressen und

- Wassertopf

Dermatom ist ein mit Akku oder Luftdruck betriebenes Hautentnahmegerät, bei dem sich die Dicke der zu entnehmenden Hautstreifen in 1/10 Millimeter-Schritten einstellen lässt. Wir verwenden es sowohl für das Debridement als auch zur Entnahme von Spalthaut für den Hautersatz. Mit Weck- und Humbymesser lassen sich breitere und dickere Hautschichten abtragen.
- 1 Hegarnadelhalter mit Hautnaht (5-0-Ethilon)

- 1 Material-/Fadenschere

Darunter:

- 2 Scharfe Löffel (groß und klein)

- 1 monopolarer Elektrokauter

Unten von links nach rechts:

- 1 Jelonet-Gaze zum Einfetten der Entnamestelle

- 1 Akku-Dermatom

- 1 Hautwalze mit Trägerplatte

\section{Einmalmaterial}

- 1 Extremitäten- oder Rumpfabdeckung

Ist eine gute Blutung der obersten Schicht erreicht, stillen wir diese mit warmen, feuchten Bauchtüchern. Die Bauchtücher werden nach kurzer Einwirkzeit vorsichtig abgenommen und das OP-Gebiet gesäubert. Sind auch nach dem Debridement die Wundverhältnisse noch unklar oder die zu transplantierenden Areale zu groß, verwenden wir temporäre Hauter-
- 1 Instrumententischbezug

- 1 Basistuch

- 3 Mäntel

- Handschuhe

- 12 Abwaschtupfer

- 20 Kompressen

- 1 Jelonet $10 \times 10$

- 1 10-er Skalpell

- 1 5-0 Ethilon Hautfaden

\section{Für den Verband}

- Jelonet-Gaze

- Lavanid-Gel

- Kompressen

- Elastomull-Binden

satzmaterialien, z.B. Xenograft, und legen breite Streifen Jelonet-Gaze auf, die als weitere Infektionsprophylaxe reichlich mit Lavanid-Gel bestrichen werden. Das Ganze wird in große Mullkompressen eingeschlagen, für Thorax und Rücken benutzen wir Mullauflagen in Westenform. Der äußere Verband besteht je nach Blutungsstärke aus Mull- oder elastischen Binden. 
Eine weitere Möglichkeit ist der Vakuumverband, der zu einer vermehrten Granulationsbildung führt und sich gut als Vorbereitung für die spätere Hauttransplantation eignet. Hierfür verwenden wir schwarze Vacusealschwämme, die der Wundfläche entsprechend zurechtgeschnitten und auf den Defekt gelegt werden. Sie werden mit durchsichtigen sterilen Klebefolien luftdicht aufgeklebt. Ein Saugnippel wird angebracht und durch ein Schlauchsystem vom Springer mit der unsterilen elektrischen Pumpe verbunden. Ein gleichmäßiger Unterdruck saugt das Wundsekret $\mathrm{ab}$ und regt die Bildung von Granulationsgewebe an.

INFO Vor dem Anlegen der Folie empfiehlt es sich, die Haut mit Benzin zu entfetten. Trotzdem ist es, vor allem bei unebenen Flächen, Hautfalten oder im Bereich von Fingern oder Zehen oft schwierig, ein wirklich dichtes Vakuum zu erzeugen. Im Bereich der Hände ist eventuell die Verwendung von speziellen Handschuhen sinnvoll ( $\rightarrow$ Abb. 7).

Ist der Patient kreislaufstabil und ein guter Wundgrund vorhanden, kann eine Spalthauttransplantation durchgeführt werden ( $\rightarrow$ Abb. 8 zeigt gut eingewachsene Spalthaut). Wir benötigen ein Dermatom mit Hautwalze und verschiedene Trägerplatten (1:1,5, 1:3 und 1:6), bei Bedarf auch eine Meek-Walze. Der Springer schließt das Dermatom an die Druckluft an oder bestückt es mit einem Akku. Der Instrumentierende legt eine sterile Klinge ein und stellt die Schnitttiefe auf $1 / 3 \mathrm{~mm}$ ein. Die Hautentnahme erfolgt an einer gesunden Stelle, z.B. Oberschenkel, Oberarm oder Rücken. Die Entnahmestelle wird eingefettet (wir benutzen hierfür das Deckblatt einer Jelonet-Wundauflage), und der Assistent spannt die Haut zur besseren Abnahme. Während der Chirurg die Haut entnimmt, zieht der Instrumentierende vorsichtig mit zwei atraumatischen Pinzetten an der austretenden Haut, um ein Zurückrutschen des Hautstreifens ins Dermatommesser zu verhindern ( $\rightarrow$ Abb. 9). Auf die Entnahmestelle wird eine aufgefaltete Kompresse oder Fettgaze gelegt und in der Regel bis zur Spontanregeneration belassen.
Der Instrumentierende streicht die Haut für das Mesh-graft auf einer befeuchteten oder eingefetteten Trägerplatte vorsichtig glatt, bis die Ränder nicht mehr eingerollt sind, und dreht die Trägerplatte langsam durch die Walze ( $\rightarrow$ Abb. 10). Der Chirurg schneidet die gemeshte Haut $(\rightarrow$ Abb. 11) mit einer feinen Schere dem Defekt entsprechend zu, klammert sie fest oder näht sie mit nicht resorbierbarem Faden ein.

\section{INFO Hautreste feucht halten und aufbewahren! Auch kleine Reste kön- nen bei entsprechender Dehnung noch größere Defekte decken und er- sparen dem Patienten eine weitere, schmerzhafte Entnahmestelle.}

Haben wir die Möglichkeit, Trägerplatten mit geringem Vergrößerungsfaktor $(1: 1,5)$ zu benutzen, ist das kosmetische Ergebnis gut. Bei großflächigen Verbrennungen kann mit einer entsprechenden Trägerplatte (1:6) viel Fläche gewonnen werden, das kosmetische Ergebnis ist jedoch deutlich schlechter. Ein Maximum an Flächengewinn (1:12) bringt das Meek-Micrografting ( $\rightarrow$ Abb. 12). Hier wird die Haut auf einer Korkplatte in kleine Würfel von $3 \times 3 \mathrm{~mm}$ geschnitten und anschließend auf ein Gewebeplissee geklebt. Dies ist, wie auch seine Trägerfolie aus Aluminium, in zwei Richtungen vorgefaltet. Die Fältelungen werden vorsichtig auseinander gezogen und das Transplantat auf den Defekt aufgebracht. Die Alufolie wird entfernt, überschüssige Trägersubstanz abgeschnitten und die verbleibende festgeklammert. Sie kann nach 6 Tagen entfernt werden. Ist auch ein Micrografting nicht ausreichend, können mit einer minimalen Hautentnahme Keratinozyten-Kulturen angelegt werden. Dies ist jedoch zeitaufwändig und die entstandene Zuchthaut ist empfindlich und infektanfällig.

Für den Verband verwenden wir ganz aufgefaltete Kompressen, die wolkenartig auf das Transplantat aufgewickelt werden und so das Einwachsen des Hauttransplantates durch leichten Druck unterstützen. Sie werden mit elastischen Mullbinden angewickelt.

\section{Wissenswertes}

Behandlungskonzepte für Verbrennungen sind schon seit $1500 \mathrm{v}$. Chr. schriftlich belegt, die Einteilung in Verbrennungsgrade seit 1600 n. Chr. 1869 unternahm Reverdin erste Versuche, Haut zu transplantieren, und um 1900 erkannte man auch die Bedeutung des Flüssigkeitsverlustes für den Verbrannten. Die heute übliche Methode der sofortigen Abtragung des untergegangenen Gewebes und der Deckung mit Spalthaut wird seit 1968 durchgeführt. Doch erst heute ermöglicht das Zusammenspiel von verbessertem intensivmedizinischem Management des Schockgeschehens und operativer Deckung der Wundfläche einem Patient mit einer zu 22\% verbrannten Körperoberfläche eine Überlebenschance von $80,1 \%$.

\section{Literatur}

Bruck, Müller, Stehen: Handbuch der Verbrennungstherapie. Ecomed, Landsberg 2002

Henckel von Donnersmarck: Verbrennungen. W. Zuckschwerdt, München 1990

Herzog, Reul, Jenninger: Verbrennungen. Kohlhammer, Stuttgart 1989

Lorenz S, Zellner P: Die Infektion beim Brandverletzten. Steinkopff, Darmstadt 1993

Pallua, von Bülow: Behandlungskonzepte bei Verbrennungen. In: Der Chirurg 2006/2, Springer Medizinverlag Hannover

Hartmann, Ottomann: Flüssigkeits-, Schmerz- und chirurgische Therapie des Verbrennungstraumas in den ersten Stunden nach Klinikaufnahme. In: Notfall- und Rettungsmedizin 2010/1, Springer Medizinverlag

\begin{tabular}{l}
\hline AUTOREN \\
Irene Jantzen \\
Fachschwester für den \\
Operationsdienst \\
Klinik für Plastische, Hand-, \\
Rekonstruktive und Verbrennungschirurgie an der \\
Eberhard Karls Universität Tübingen / BG Unfallklinik \\
nenajantzen@web.de
\end{tabular}

Dr. med. A. Rahmanian-Schwarz Oberarzt

Klinik für Plastische, Hand-, Rekonstruktive und Verbrennungschirurgie an der Eberhard Karls Universität Tübingen / BG Unfallklinik

ARahmanian@bgu-tuebingen.de

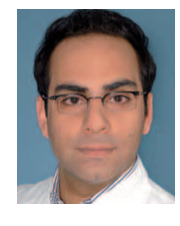

BIBLIOGRAFIE

DOI 10.1055/s-0031-1284309

Im OP 2011; 5: 204-207

(c) Georg Thieme Verlag KG

Stuttgart . New York . ISSN 1611-7905 